\title{
Neutrophils associate with Bowman's capsule rupture specifically in PR3-ANCA glomerulonephritis
}

\author{
Samy Hakroush ${ }^{1} \cdot$ Björn Tampe ${ }^{2}$ (1)
}

Received: 8 September 2021 / Accepted: 9 November 2021 / Published online: 1 December 2021

(c) The Author(s) 2021

\begin{abstract}
Background Renal involvement is a common and severe complication of ANCA (antineutrophil cytoplasmic antibody) associated vasculitis (AAV) potentially resulting in a pauci-immune necrotizing and crescentic antineutrophil cytoplasmic antibody (ANCA) glomerulonephritis (GN) with acute kidney injury (AKI), end-stage renal disease (ESRD) or death. We recently described that Bowman's capsule rupture links glomerular damage to tubulointerstitial inflammation in ANCAassociated glomerulonephritis. Herein we provide a comprehensive histological subtyping of immune cell infiltrates in association with Bowman's capsule rupture in ANCA GN.

Methods A total of 44 kidney biopsies with ANCA GN were retrospectively included in a single-center observational study. Within a renal biopsy specimen, each glomerulus was scored separately for the presence of extensive and focal Bowman's capsule rupture in injured glomeruli. Infiltrates of neutrophils, eosinophils, plasma cells, and mononucleated cells (macrophages, lymphocytes) were quantified as a fraction of the area of total cortical inflammation.

Results Extensive Bowman's capsule rupture was associated with tubulointerstitial inflammation containing infiltrates of neutrophils, eosinophils and plasma cells. A similar association was observed for the presence of focal Bowman's capsule rupture, correlating with tubulointerstitial inflammation containing neutrophils, eosinophils and plasma cells. Multiple logistic regression confirmed that extensive Bowman's capsule rupture correlated with tubulointerstitial inflammation containing neutrophils, and focal Bowman's capsule rupture correlated with neutrophil and plasma cell infiltration. Furthermore, this association was specifically observed in PR3-ANCA GN.

Conclusion To our knowledge, this is the first report linking Bowman's capsule rupture directly to tubulointerstitial inflammation by immune cell subtypes. This underscores a pathomechanistic link between tubulointerstitial and glomerular lesions in ANCA GN and needs further investigation.
\end{abstract}

Björn Tampe

bjoern.tampe@med.uni-goettingen.de

1 Institute of Pathology, University Medical Center Göttingen, Göttingen, Germany

2 Department of Nephrology and Rheumatology, University Medical Center Göttingen, Göttingen, Germany 


\section{Graphical abstract}

\section{Neutrophils associate with Bowman's capsule rupture specifically in PR3-ANCA glomerulonephritis}

Methods
- 44 kidney biopsies with
confirmed ANCA
glomerulonephritis (GN) were
included in a single-center
observational study.
- Within a renal biopsy specimen,
each glomerulus was scored
separately for the presence of
extensive and focal Bowman's
capsule rupture (BCR).
- Infiltrates of neutrophils,
eosinophils, plasma cells, and
mononucleated cells
(macrophages, lymphocytes)
were quantified as a fraction of
the area of total cortical
inflammation.

Results

Extensive BCR was associated with tubulointerstitial inflammation containing infiltrates of neutrophils, eosinophils and plasma cells, a comparable association was observed for presence of focal BCR. Multiple logistic regression confirmed that tubulointerstitial inflammation containing neutrophils was associated with BCR, specifically in PR3-ANCA GN.

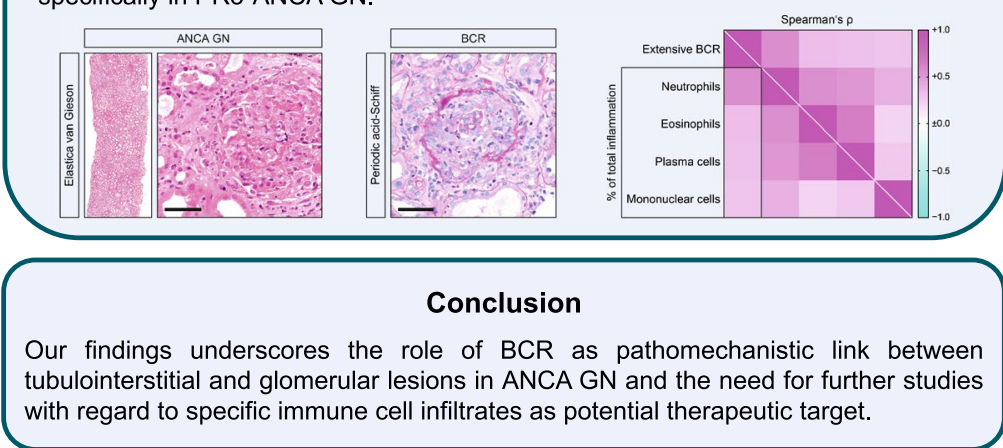

Keywords ANCA glomerulonephritis $\cdot$ Bowman's capsule rupture $\cdot$ Tubulointerstitial inflammation $\cdot$ Neutrophils

\section{Introduction}

Antineutrophil cytoplasmic antibody (ANCA)-associated vasculitis (AAV) is a small vessel vasculitis according to the 2012 revised Chapel Hill Consensus Conference Nomenclature of Vasculitides, most frequently presenting as microscopic polyangiitis (MPA) or granulomatosis with polyangiitis (GPA) [1,2]. Renal involvement is a common and severe complication of AAV potentially resulting in pauci-immune necrotizing and crescentic ANCA glomerulonephritis (GN) with acute kidney injury (AKI), end-stage renal disease (ESRD) or death [2]. Clinicopathologic studies of the European Vasculitis Study Group (EUVAS) demonstrated that distinct glomerular lesions are related to renal outcome in ANCA GN [3-6]. Derived from these studies, histopathological subgrouping into four classes (focal, crescentic, mixed, and sclerotic) as defined by Berden et al. was shown to predict long-term renal survival rates [7]. However, multivariable analyses demonstrated no improvement in outcome prediction in most of these studies, mainly attributed to no outcome difference in the crescentic and mixed classes [8-16]. Therefore, Brix et al. suggested the ANCA renal risk score (ARRS) by incorporating tubular atrophy/interstitial fibrosis (TA/IF) to the percentage of normal glomeruli and baseline glomerular filtration rate (GFR) to predict ESRD in patients with $\mathrm{AAV}$, underscoring a pathomechanistic link between tubulointerstitial and glomerular lesions in ANCA GN [17]. Bowman's capsule rupture was first described more than 30 years ago and we recently described that Bowman's capsule rupture links glomerular damage to tubulointerstitial inflammation in ANCA-associated glomerulonephritis in a considerable subset of patients with ANCA GN [18]. An increased fraction of glomeruli affected by extensive Bowman's capsule rupture in ANCA GN was associated with tubulointerstitial inflammation, suggesting that interstitial inflammation may also have predictive value in assessing the risk of decline in kidney function in ANCA GN $[4,10,19]$. Furthermore, we and others have previously described that focal Bowman's capsule rupture with less extensive lesions was observed even more frequently in ANCA GN [20, 21]. The concept that tubulointerstitial injury mediates impairment of renal function was described more than five decades ago, in studies showing that decline of kidney function exhibited a stronger correlation with the severity of tubulointerstitial rather than with glomerular damage [22]. We recently characterized intrarenal subtypes of immune cell infiltrates in myeloperoxidase (MPO)-ANCA versus proteinase 3 (PR3)-ANCA GN, associated with distinct glomerular and tubulointerstitial lesions [23]. However, the association between Bowman's capsule rupture and distinct immune cell subtypes has not been explored so far. Therefore, herein we provide comprehensive histological subtyping of immune cell infiltrates in association with extensive or focal Bowman's capsule rupture in a cohort of 44 patients with ANCA GN confirmed by renal biopsy. The cohort comprises cases who underwent a kidney biopsy between 2015 and 2020 in 
a single-center, and this study represents a further step in the description of the relationship between Bowman's capsule rupture and kidney damage [18].

\section{Methods}

\section{Study population}

A total of 44 kidney biopsies with confirmed renal involvement of ANCA GN at the University Medical Center Göttingen were retrospectively included between 2015 and 2020, the patient cohort has been previously described [18]. All studies involving human participants were reviewed and approved by the Institutional Review Board of the University Medical Center Göttingen, Germany (protocol numbers 22/2/14 and 28/09/17). Medical records were used to obtain data on age, sex, diagnosis (GPA or MPA) and laboratory results.

\section{Renal histopathology}

A renal pathologist evaluated all biopsies and was blinded to all clinical data and analyses. Within a renal biopsy specimen, each glomerulus was scored separately for the presence of extensive and focal Bowman's capsule rupture in injured glomeruli (crescentic and/or necrotic). Consequently, the percentage of glomeruli affected by Bowman's capsule rupture was calculated as a fraction of the total number of glomeruli in each renal biopsy. Infiltrates of neutrophils, eosinophils, plasma cells, and mononucleated cells (macrophages, lymphocytes) were quantified as described previously [23]. The area of total cortical inflammation was quantified in each section stained with hematoxylin-eosin (HE) as a fraction of the total area. Distinct immune cell infiltrates were quantified as follows: neutrophils, eosinophils, plasma cells, and mononucleated cells (macrophages, lymphocytes) were identified by morphological criteria and fractions of the total cortical inflammation were estimated for each cell type.

\section{Statistical methods}

Variables were tested for normal distribution using the Shapiro-Wilk test. Statistical comparisons were not formally powered or prespecified. Spearman correlation analyses were used to analyze correlations and shown by a heatmap reflecting mean values of Spearman's $\rho$, asterisks indicate $p<0.05$. Data analyses were performed with GraphPad Prism (version 8.4.3 for MacOS, GraphPad Software, San Diego, California, USA). Multiple regression analyses were performed using IBM SPSS Statistics (version 27 for MacOS, IBM Corporation, Armonk, NY, USA). A probability $(p)$ value of $<0.05$ was considered statistically significant.

\section{Results}

Tubulointerstitial inflammation in ANCA GN contained neutrophils, eosinophils, plasma and mononuclear cells (macrophages and lymphocytes, Fig. 1A). Extensive Bowman's capsule rupture was associated with tubulointerstitial inflammation containing infiltrates of neutrophils, eosinophils and plasma cells (Fig. 1B, C). In contrast, infiltration of mononuclear cells including macrophages and lymphocytes did not show an association with Bowman's capsule rupture in ANCA GN (Fig. 1C). A similar association was observed for the presence of focal Bowman's capsule rupture, correlating with tubulointerstitial inflammation containing neutrophils, eosinophils and plasma cells (Fig. 1D). Multiple logistic regression confirmed that extensive Bowman's capsule rupture was associated with tubulointerstitial inflammation containing neutrophils ( $p<0.01$, Table 1$)$, and that focal Bowman's capsule rupture was associated with neutrophil $(p<0.01)$ and plasma cell infiltrates $(p<0.05$, Table 2). To elucidate the contribution of immune cell infiltration to Bowman's capsule rupture in distinct ANCA subtypes, we then analyzed PR3-ANCA and MPO-ANCA GN separately. Interestingly, an association between tubulointerstitial inflammation containing neutrophils and Bowman's capsule rupture was specifically observed in PR3-ANCA GN (Fig. 2A). In contrast, no association between subtypes of immune cell infiltrates and Bowman's capsule rupture was observed in MPO-ANCA GN (Fig. 2B).

\section{Discussion}

The performance of the established histopathological subtyping proposed by Berden et al. and Brix et al. has improved with the implementation of Bowman's capsule rupture to the classification systems [24]. The additional predictive value of Bowman's capsule rupture is linked to its marker of irreversible nephron damage, as a consequence of segmental glomerulosclerosis caused by Bowman's capsule rupture.

Moreover, an increased of glomeruli showing Bowman's capsule rupture in ANCA GN was associated with tubulointerstitial inflammation, further stressing the predictive value of interstitial inflammation in the decline of kidney function in ANCA GN [4, 10, 19].

In addition, we recently characterized intrarenal subtypes of immune cell infiltrates in MPO-ANCA versus PR3-ANCA $\mathrm{GN}$, associated with distinct glomerular and tubulointerstitial lesions [23]. Based on these previous observations, we here aimed to analyze the association between Bowman's 
A

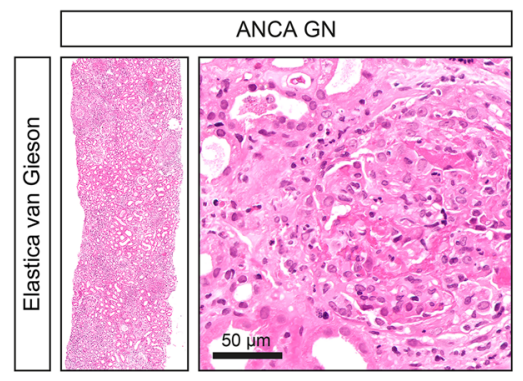

B

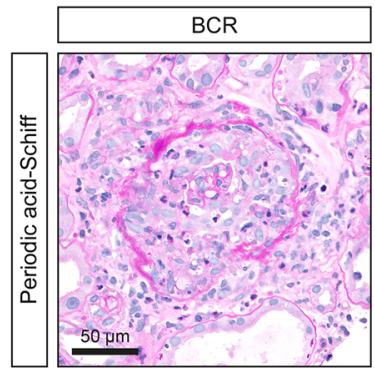

C

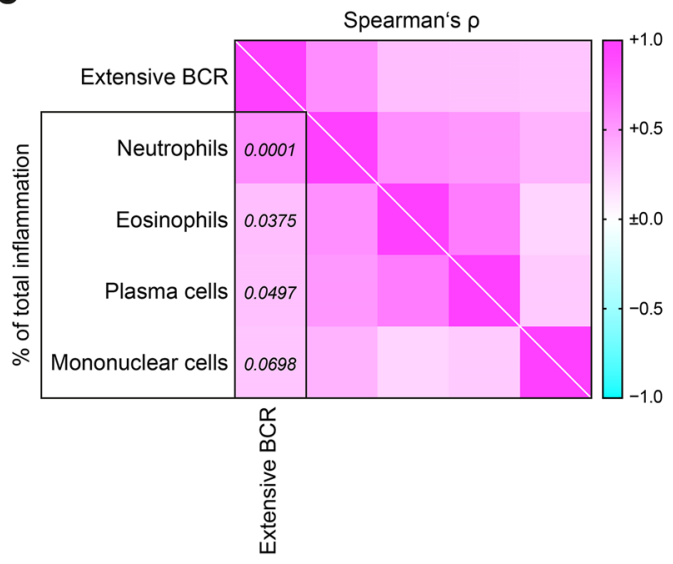

D

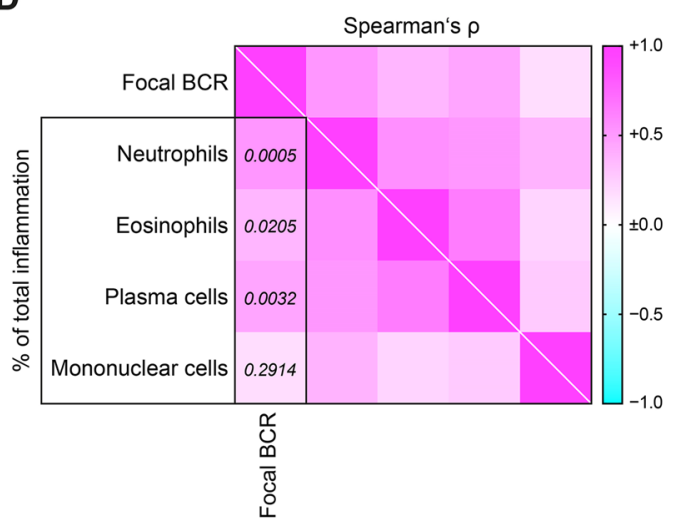

Fig. 1 Tubulointerstitial inflammation containing neutrophils associates with BCR in ANCA GN. A Representative Elastica van Gieson-stained renal biopsy in ANCA GN (scale bar: $50 \mu \mathrm{m}$ ). B Representative periodic acid-Schiff reaction-stained renal biopsy in ANCA GN with BCR (scale bar: $50 \mu \mathrm{m}$ ). C, D The association between the fraction of glomeruli affected by extensive or focal BCR, and sub-

Table 1 Multiple regression analysis for immune cell infiltrates associated with extensive BCR in ANCA GN

\begin{tabular}{lrll}
\hline Immune cell subtype & \multicolumn{1}{l}{$\beta$} & SE & p value \\
\hline Neutrophils_\% & 0.4941 & 1.4642 & $\mathbf{0 . 0 0 5 6}$ \\
Eosinophils_\% & 0.2529 & 2.0512 & 0.2440 \\
Plasma cells_\% & -0.1531 & 0.5061 & 0.4499 \\
Mononucleated cells_\% & -0.1075 & 0.2692 & 0.4866 \\
\hline
\end{tabular}

$A N C A$ anti-neutrophil cytoplasmic antibodies, BCR Bowman's capsule rupture, $G N$ glomerulonephritis, $S E$ standard error

Bold indicates statistically significant value

capsule rupture and distinct immune cell infiltrates in ANCA GN. To our knowledge, this is the first report linking Bowman's capsule rupture directly to tubulointerstitial inflammation by characterizing immune cell subtypes. types of immune cell infiltrates in ANCA GN is shown by a heatmap reflecting mean values of Spearman's $\rho$, corresponding values of $p$ are shown. ANCA anti-neutrophil cytoplasmic antibodies, BCR Bowman's capsule rupture, $G N$ glomerulonephritis, $M P O$ myeloperoxidase, $P R 3$ proteinase 3

Table 2 Multiple regression analysis for immune cell infiltrates associated with focal BCR in ANCA GN

\begin{tabular}{llll}
\hline Immune cell subtype & $\beta$ & SE & $\mathrm{p}$ value \\
\hline Neutrophils_\% & 0.4921 & 3.3055 & $\mathbf{0 . 0 0 5 6}$ \\
Eosinophils_\% & -0.2345 & 4.6306 & 0.2779 \\
Plasma cells_\% & 0.4146 & 1.1425 & $\mathbf{0 . 0 4 5 1}$ \\
Mononucleated cells_\% & -0.1441 & 0.6077 & 0.3508 \\
\hline
\end{tabular}

$A N C A$ anti-neutrophil cytoplasmic antibodies, $B C R$ Bowman's capsule rupture, $G N$ glomerulonephritis, $S E$ standard error

Bold indicates statistically significant value

We observed that extensive Bowman's capsule rupture correlated with tubulointerstitial inflammation containing neutrophils, and that focal Bowman's capsule rupture correlated with neutrophil and plasma cell infiltration. This association was specifically observed in PR3-ANCA GN [25]. 
A

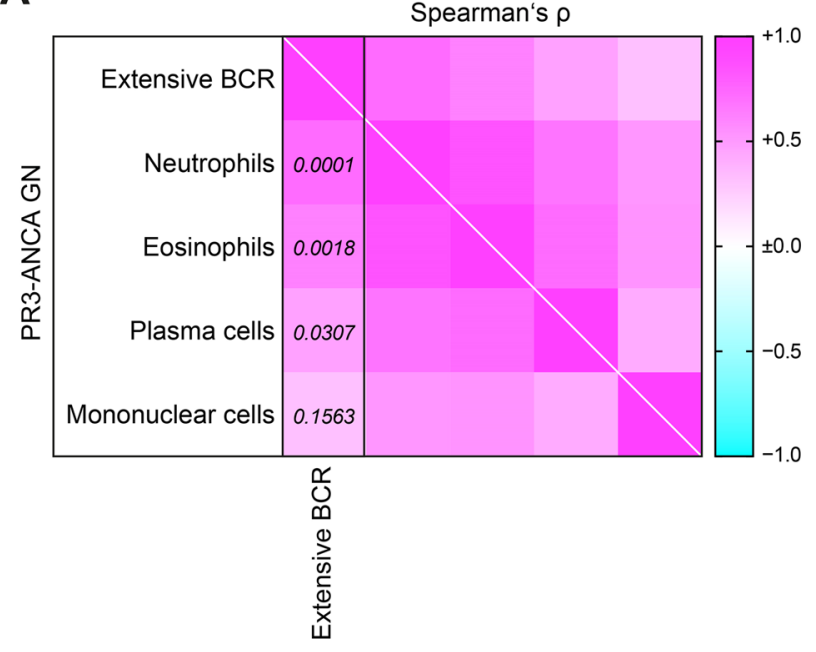

C

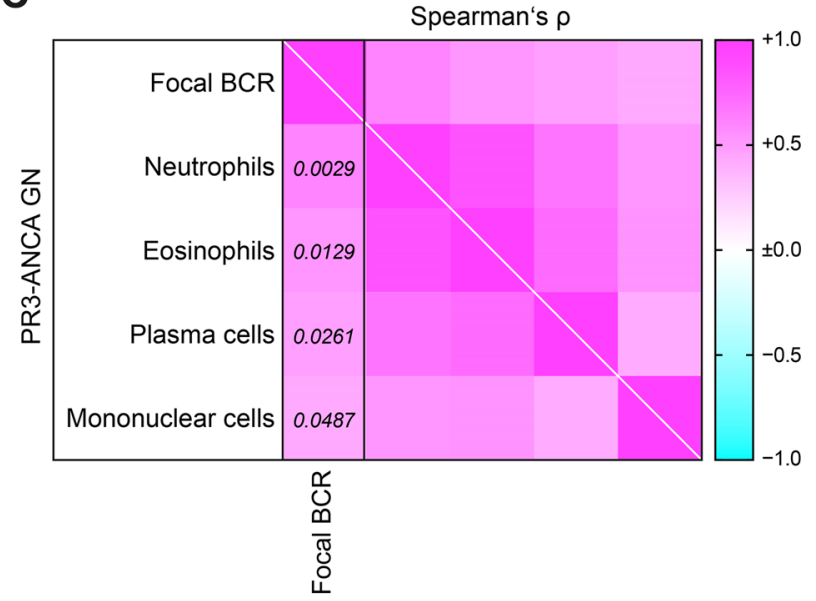

Fig. 2 Tubulointerstitial inflammation containing neutrophils associates with BCRspecifically in PR3-ANCA GN. A, B Association between the fraction of glomeruli affected by extensive BCR and subtypes of immune cell infiltrates separated for PR3-ANCA and MPOANCA GN is shown by a heatmap reflecting mean values of Spearman's $\rho$, corresponding values of $p$ are shown. C, D The association

At disease onset, neutrophils are activated by pathogenic ANCAs, causing a release of inflammatory cytokines, reactive oxygen species, and lytic enzymes, thus resulting in an excessive formation of neutrophil extracellular traps (NETs) [26]. Intrarenal infiltrates of primed neutrophils are activated by MPO-ANCA and PR3ANCA, leading to degranulation with release of cytoplasmic granules into the glomerular and interstitial space. As a result, reactive oxygen radicals (RORs) accumulate and cause vascular damage [26]. In addition, stimulation of neutrophils causes the release of factors that activate the alternative complement system, aggravating vascular damage [26]. Small vessel leakage of serum proteins and the formation of fibrin give rise to fibrinoid necrosis. Besides
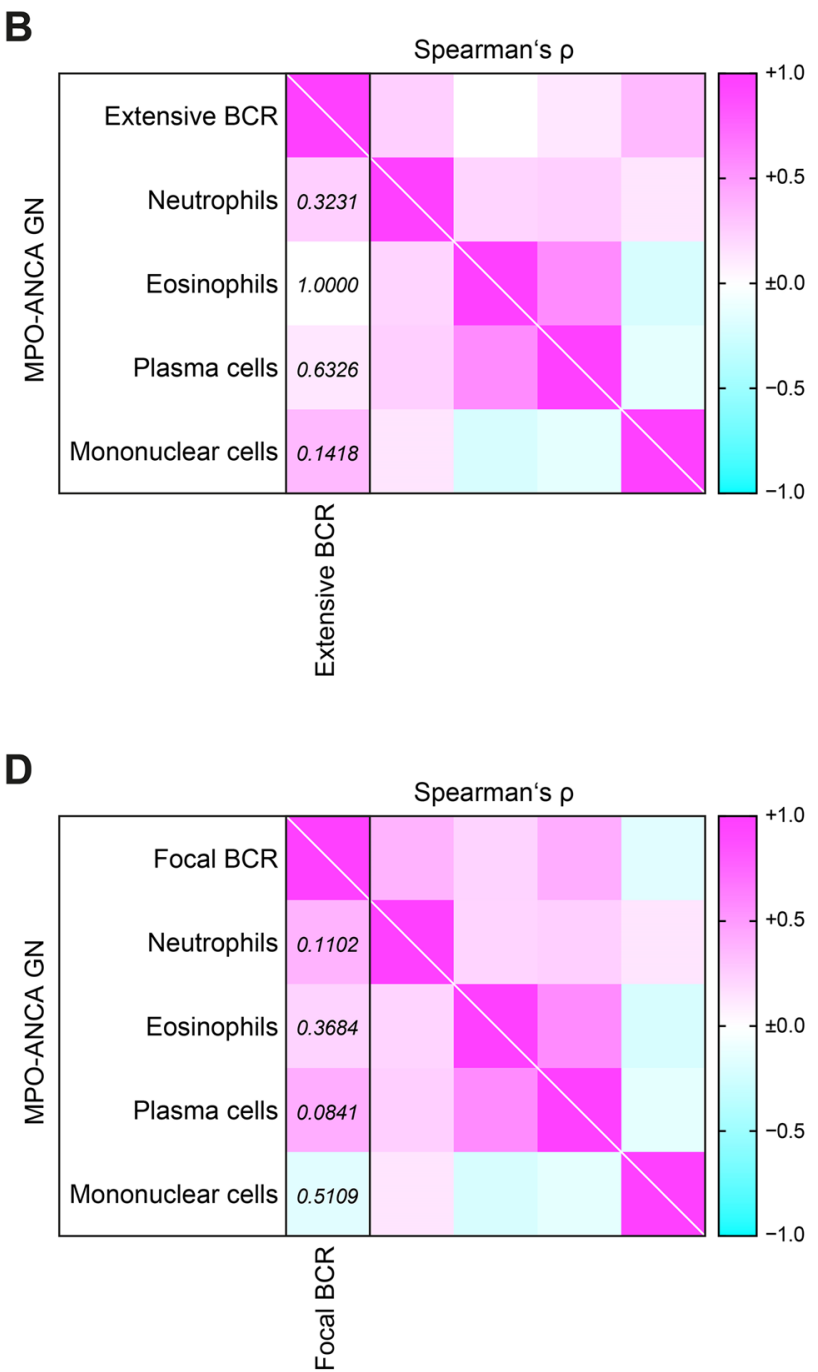

between the fraction of glomeruli affected by focal BCR and subtypes of immune cell infiltrates separated for PR3-ANCA and MPO-ANCA GN is shown by a heatmap reflecting mean values of Spearman's $\rho$, corresponding values of $p$ are shown. ANCA anti-neutrophil cytoplasmic antibodies, $B C R$ Bowman's capsule rupture, $G N$ glomerulonephritis, $M P O$ myeloperoxidase, $P R 3$ proteinase 3

neutrophils, plasma cell infiltration is also commonly observed in ANCA GN and has been associated with tubulointerstitial inflammation and IgG4 positivity [27]. While neutrophil and plasma cell infiltrates predominate the early phase of inflammation in AAV, necrotizing lesions evolve into granuloma rich in monocyte/macrophages, and this is accompanied by infiltration of lymphocytes, such as $\mathrm{T}$ cells $[28,29]$. Our observation that an association between neutrophil infiltration and Bowman's capsule rupture was specifically seen in PR3-ANCA GN suggests distinct pathogenetic mechanisms in ANCA subtypes, requiring further investigation.

The main limitations of our study are its retrospective design in a single center and a selection bias towards more 
severe cases of ANCA GN with limited information on kidney function before the kidney biopsy.

Nevertheless, Bowman's capsule rupture has independently been associated with poor renal outcome in ANCA GN [24].

This underscores the need for further studies with regard to specific immune cell infiltrates as a potential therapeutic target in distinct subtypes of severe ANCA GN.

Author contributions $\mathrm{SH}$ and BT conceived the study, collected and analyzed data and co-wrote the manuscript.

Funding Open Access funding enabled and organized by Projekt DEAL. This publication was supported financially by the Open Access Grant Program of the German Research Foundation (DFG) and the Open Access Publication Fund of the University of Göttingen. BT was also supported by the Research program, University Medical Center, University of Göttingen (1403720). The funding sources were not involved in the design, collection, analysis, interpretation, writing or decision to submit the article.

Data availability Deidentified data are available on reasonable request from the corresponding author.

\section{Declarations}

Conflict of interest Each author certifies that he, or a member of their immediate family, has no commercial associations (e.g., consultancies, stock ownership, equity interest, patent/licensing arrangements, etc.) that might pose a conflict of interest in connection with the contents of the submitted article. The results presented in this article have not been published previously in whole or part, except in abstract format.

Ethics approval All studies involving human participants were reviewed and approved by the Institutional Review Board of the University Medical Center Göttingen, Germany (protocol numbers 22/2/14 and 28/09/17).

Open Access This article is licensed under a Creative Commons Attribution 4.0 International License, which permits use, sharing, adaptation, distribution and reproduction in any medium or format, as long as you give appropriate credit to the original author(s) and the source, provide a link to the Creative Commons licence, and indicate if changes were made. The images or other third party material in this article are included in the article's Creative Commons licence, unless indicated otherwise in a credit line to the material. If material is not included in the article's Creative Commons licence and your intended use is not permitted by statutory regulation or exceeds the permitted use, you will need to obtain permission directly from the copyright holder. To view a copy of this licence, visit http://creativecommons.org/licenses/by/4.0/.

\section{References}

1. Jennette JC et al (2013) 2012 revised International Chapel Hill consensus conference nomenclature of vasculitides. Arthritis Rheum 65(1):1-11
2. Hruskova $\mathrm{Z}$ et al (2015) Characteristics and outcomes of granulomatosis with polyangiitis (Wegener) and microscopic polyangiitis requiring renal replacement therapy: results from the European Renal Association-European Dialysis and Transplant Association Registry. Am J Kidney Dis 66(4):613-620

3. Bajema IM et al (1999) Kidney biopsy as a predictor for renal outcome in ANCA-associated necrotizing glomerulonephritis. Kidney Int 56(5):1751-1758

4. Hauer HA et al (2002) Determinants of outcome in ANCA-associated glomerulonephritis: a prospective clinico-histopathological analysis of 96 patients. Kidney Int 62(5):1732-1742

5. de Lind van Wijngaarden RA et al (2006) Clinical and histologic determinants of renal outcome in ANCA-associated vasculitis: a prospective analysis of 100 patients with severe renal involvement. J Am Soc Nephrol 17(8):2264-2274

6. Bajema IM et al (1996) The renal histopathology in systemic vasculitis: an international survey study of inter- and intra-observer agreement. Nephrol Dial Transplant 11(10):1989-1995

7. Berden AE et al (2010) Histopathologic classification of ANCA-associated glomerulonephritis. J Am Soc Nephrol 21(10): $1628-1636$

8. Hilhorst $\mathrm{M}$ et al (2013) Estimating renal survival using the ANCA-associated GN classification. J Am Soc Nephrol 24(9):1371-1375

9. Iwakiri $\mathrm{T}$ et al (2013) Validation of a newly proposed histopathological classification in Japanese patients with anti-neutrophil cytoplasmic antibody-associated glomerulonephritis. BMC Nephrol 14:125

10. Ford SL et al (2014) Histopathologic and clinical predictors of kidney outcomes in ANCA-associated vasculitis. Am J Kidney Dis 63(2):227-235

11. Quintana LF et al (2014) ANCA serotype and histopathological classification for the prediction of renal outcome in ANCA-associated glomerulonephritis. Nephrol Dial Transplant 29(9):1764-1769

12. Moroni $G$ et al (2015) Predictors of renal survival in ANCAassociated vasculitis. Validation of a histopatological classification schema and review of the literature. Clin Exp Rheumatol 33(2 Suppl 89):S56-63

13. Andreiana I et al (2015) ANCA positive crescentic glomerulonephritis outcome in a Central East European cohort: a retrospective study. BMC Nephrol 16:90

14. Tanna A et al (2015) Long-term outcome of anti-neutrophil cytoplasm antibody-associated glomerulonephritis: evaluation of the international histological classification and other prognostic factors. Nephrol Dial Transplant 30(7):1185-1192

15. van Daalen E et al (2015) Twenty-five years of RENHIS: a history of histopathological studies within EUVAS. Nephrol Dial Transplant 30(Suppl 1):i31-i36

16. Bjorneklett R, Sriskandarajah S, Bostad L (2016) Prognostic value of histologic classification of ANCA-associated glomerulonephritis. Clin J Am Soc Nephrol 11(12):2159-2167

17. Brix SR et al (2018) Development and validation of a renal risk score in ANCA-associated glomerulonephritis. Kidney Int 94(6):1177-1188

18. Hakroush S et al (2021) Bowman's capsule rupture links glomerular damage to tubulointerstitial inflammation in ANCAassociated glomerulonephritis. Clin Exp Rheumatol 39(2 Suppl 129):27-31

19. Hakroush $\mathrm{S}$ et al (2021) Systematic histological scoring reveals more prominent interstitial inflammation in myeloperoxidaseANCA compared to proteinase 3-ANCA glomerulonephritis. J Clin Med 10(6):1231

20. Hakroush S, Tampe B (2021) Correspondence on 'Bowman's capsule rupture on renal biopsy improves the outcome prediction of 
ANCA-associated glomerulonephritis classifications. Ann Rheum Dis

21. L'Imperio V et al (2021) Response to: 'Correspondence on 'Bowman's capsule rupture on renal biopsy improves the outcome prediction of ANCA-associated glomerulonephritis classifications" by Hakroush and Tampe'. Ann Rheum Dis

22. Risdon RA, Sloper JC, De Wardener HE (1968) Relationship between renal function and histological changes found in renalbiopsy specimens from patients with persistent glomerular nephritis. Lancet 2(7564):363-366

23. Hakroush S et al (2021) Comparative histological subtyping of immune cell infiltrates in MPO-ANCA and PR3-ANCA glomerulonephritis. Front Immunol 12:737708

24. L'Imperio V et al (2020) Bowman's capsule rupture on renal biopsy improves the outcome prediction of ANCA-associated glomerulonephritis classifications. Ann Rheum Dis

25. Chen A et al (2018) Bowman's capsule provides a protective niche for podocytes from cytotoxic CD8+ T cells. J Clin Investig 128(8):3413-3424
26. Nakazawa D et al (2019) Pathogenesis and therapeutic interventions for ANCA-associated vasculitis. Nat Rev Rheumatol 15(2):91-101

27. Masuzawa $\mathrm{N}$ et al (2019) Clinicopathological analysis of ANCAassociated glomerulonephritis focusing on plasma cell infiltrate. Clin Exp Nephrol 23(12):1373-1381

28. Jennette JC et al (2013) Pathogenesis of antineutrophil cytoplasmic autoantibody-associated small-vessel vasculitis. Annu Rev Pathol 8:139-160

29. Jennette JC, Falk RJ (2014) Pathogenesis of antineutrophil cytoplasmic autoantibody-mediated disease. Nat Rev Rheumatol 10(8):463-473

Publisher's Note Springer Nature remains neutral with regard to jurisdictional claims in published maps and institutional affiliations. 